\title{
De George Sand à Henry Murger : note sur les débuts du bohémianisme (1835-1845)
}

\author{
Françoise Genevray \\ Université Jean Moulin-Lyon 3
}

La décennie 1835-1845 sépare l'éclosion de la bohème comme motif littéraire ${ }^{1}$ et sa canonisation par Henry Murger. Longtemps, l'homophonie entre bohème et Bohême, doublée d'une synonymie entre bohème et bohémien, contribue à enchevêtrer ces notions. Le substantif bohême désigne au Moyen Âge un vagabond étranger, à peu près le bohémien d'aujourd'hui ${ }^{2}$. Une signification sociale apparaît au XVIIe siècle quand il catalogue un individu réfractaire aux règles de la vie

\footnotetext{
1 Décalée d'une réalité sociologique préexistante : voir Goulemot et Oster.

${ }^{2}$ Le Gitan ou Tzigane passe pour originaire d'Égypte (gipsy, zingaro), puis de Bohême.
} 
commune. Les Scènes de la vie de Bohème contribuent à fixer le sens maintenant reçu de bohème, qui perdra peu à peu son circonflexe ${ }^{3}$. Pourtant, Murger lui-même utilise indifféremment bohème ou bohémien ${ }^{4}$, comme beaucoup de ses contemporains.

L'intérêt du doublet bohème-bohémien dépasse le cadre de la pure lexicologie pour s'étendre à l'histoire culturelle et littéraire. Parus de 1845 à 1849 dans Le Corsaire-Satan, adaptés à la scène (1849) et réunis en recueil (1851), les feuilletons de Murger forgent le paradigme de la bohème comme milieu et mode de vie des jeunes artistes pauvres. Leur succès déclencha un flot de publications s'attachant à la dépeindre, à cerner sa nature et ses contours. Mais si « la notion de bohème ne fut pas mise au point et demeura sporadique jusqu'aux abords de 1850 , où elle prit une place plus nette et plus durable dans la conscience française » (Seigel, p. 37), c'est entre autres raisons parce qu'il lui fallut du temps pour se dégager du bohémien. Figure ancienne et quasiment mythique, ce dernier, loin de céder la place, fait puissamment écho aux aspirations romantiques. Les membres du Petit Cénacle (1830-1832) s'identifient aux «Bohémiens sans toits, sans bancs, / sans existence engainée » (cité par Laisney, p. 303) pour afficher leur rupture avec les codes de vie bourgeois et avec l'esthétique néo-classique. Stigmatisé par l'opinion courante ou valorisé par l'esprit romantique, le bohémien nuit durablement à la visibilité du bohème.

Examiner la démarcation entre cénacles et bohème

\footnotetext{
${ }^{3}$ Longtemps l'homophonie entre bohème et bohême fait hésiter sur le choix de l'accent. Murger lui-même utilise parfois le circonflexe dans son manuscrit, et l'hésitation continue après lui.

4 Voir notamment sa « Préface » (mai 1850) aux Scènes de la vie de bohème.
} 
n'entre pas dans notre propos, tout en appelant une remarque qui s'y rattache. Le lien de filiation établi après coup par T. Gautier entre la bohème et le Petit Cénacle constitue certes un anachronisme. Mais que l'ombre du bohémien continue de hanter toute évocation de la bohème artiste n'est pas moins évident. Nerval, qui fut mêlé au Petit Cénacle, prolonge l'ambiguïté orthographique (bohème/Bohême) et sémantique (Bohême/bohémien) en entrelaçant trois notions : la «bohême galante» de sa jeunesse au cœur du vieux Paris, l'errance bohémienne de ses vagabondages aux alentours de la capitale et la Bohême scripturale de ses « petits châteaux » imaginaires ${ }^{5}$. En 1854, un opuscule intitulé Paris-Bohème remémore la liaison primitive : «Vous avez sans doute rencontré sur les routes ces bandes de Zingari venus du pays du soleil, et qu'on appelle, je ne sais pourquoi, des bohémiens. Ces enfants de la liberté vont partout où les entraîne le caprice [...] ces gens-là sont les bohèmes de la grande route; soyons, nous, les bohèmes de la grande ville. » (Delord, p. 9)

Le bohémien-bohème des années 1830 et 1840 offre un assortiment composite: jeunesse, activités artistiques, vie instable et désordonnée, accointances avec les bas-fonds. Le dernier trait s'explique par la persistance du bohémien dans l'imaginaire de la marginalité urbaine. Les Zingari sont à la fois fascinants et suspects, de même les bohémiens. Dans leur pièce Les Bohémiens de Paris (1843), D'Ennery et Grangé les définissent comme des individus sans "asile reconnu », sans profession fixe, subsistant d'expédients plus ou moins licites, «prêts à vivre honnêtement s'ils le peuvent, et autrement s'ils

5 La Bohême Galante (1852), Les Petits Châteaux de Bohême (1853), Promenades et Souvenirs (1854-1855). 
ne le peuvent pas » (acte I, scène 7). Murger marque donc un jalon décisif en isolant la gent artiste d'une population équivoque de parasites, d'aventuriers, de charlatans et de filous. Ainsi débarrasse-t-il la bohème des éléments troubles qui ternissent l'image du bohémien, réputé chapardeur et asocial. Bien que le mélange antérieur ressurgisse parfois, la notion de bohème renverra après lui aux jeunes artistes sans le sou, qui convertissent l'âpre nécessité en liberté joyeuse.

Entre le bohémien métaphorique des amis de Pétrus Borel, Gautier ou Nerval et le bohème réputé véritable (quoique littérarisé) des Scènes de la vie de Bohème s'intercale une phase où l'artiste bohème émerge chez des personnages fictifs dits «bohémiens ", qui sont en fait des artistes pauvres analogues à ceux que peindra Murger. Cette étape intermédiaire n'a guère été observée. Réservant la référence bohémienne stricto sensu à la "préhistoire du bohémianisme», antérieure à sa "fondation véritable », Seigel évacue les années 1840, survolées en deux pages (p. 37-38) qui sautent directement de 1834 au début des années 1850. La plupart des chercheurs oublient d'inclure George Sand dans ce cadre ou s'en tiennent comme leur collègue à La dernière Aldini (1838), négligeant des textes sandiens un peu postérieurs et assez différents. Le bohémien chez Balzac et Sand appartient justement à cet intervalle sur lequel nous souhaitons arrêter l'attention. La thématique bohémienne s'accompagne en effet chez eux d'un traitement nouveau, qui l'inscrit dans un moment précis de l'Histoire. La figure de l'artiste-bohémien, jusqu'alors intemporelle, voire pour Sand quelque peu abstraite, se redéfinit pendant cette phase où nouvelle et roman prennent en charge sa représentation réaliste, situant l'individu et les groupes bohèmes dans un contexte socio-économique en pleine recomposition. 


\section{L'artiste sur le marché : Balzac, Champfleury}

«Pour les artistes et les écrivains, le changement fondamental se résume assez facilement: le mécénat céda la place au marché. » (Seigel, p. 22) Les créateurs du XIXe siècle affrontent tous peu ou prou les contraintes liées à cette mutation, dont les conséquences néfastes sont dénoncées dès l'orée des années 1830. S'il se plie au goût de ses nouveaux clients, l'artiste risque de se voir condamné à faire « des œuvres de boudoir, de salon; il livre son talent à l'industrie, aux caprices de la mode, aux fantaisies de ceux qui achètent, les bourgeois ${ }^{6}$. Pour Vigny (Chatterton, 1835), le poète n'a que des malheurs à attendre d'une société où les valeurs marchandes supplantent le goût aristocratique. Soumise au calcul des coûts et des revenus, la «littérature industrielle» (Sainte-Beuve, 1839) menace l'indépendance spirituelle de l'écrivain.

La figure du bohémien, relayée ensuite par le bohème, incarne la difficile insertion du créateur dans une société de plus en plus dominée par l'argent: les artistes "sont les Bohémiens d'aujourd'hui », lance F. Pyat en 1834. Dans «Un grand homme de province à Paris », deuxième partie d'Illusions perdues (1839), Balzac montre deux voies ouvertes au «bohémien » de la pensée ou des arts. Enivré par ses premiers succès de plume comme journaliste, Lucien de Rubempré cède aux séductions périlleuses du métier. L'épisode s'achève par un désastre professionnel, moral et financier. Quand les créanciers s'abattent sur le domicile qu'il partage avec sa maîtresse, l'actrice Coralie tombée elle aussi en disgrâce, Balzac parle de la « détresse absolue » des «deux bohémiens » dont on saisit les meubles. Loin du futur stéréotype de la bohème joyeuse et

\footnotetext{
${ }^{6}$ Lettre signée « Un artiste », L'Artiste, n IV, 1832, p. 81.
} 
désinvolte, il peint un Lucien accablé chez qui « la misère avait éteint toute fierté » (p. 448). À cette destinée pitoyable l'auteur oppose le fier Cénacle des neuf grands esprits réunis rue des Quatre-Vents dans une " froide mansarde » où se réalisent « les plus beaux rêves de sentiment». Le Cénacle comprend un interne en médecine (Bianchon), un peintre, un écrivain, un philosophe, etc., et le penseur républicain Michel Chrétien : «ce gai bohémien de l'intelligence, ce grand homme d'État, qui peutêtre eût changé la face du monde, mourut au cloître Saint-Merry comme un simple soldat». À l'époque où Lucien fait sa connaissance, Chrétien subsiste en bohème des lettres grâce à de petits travaux dans l'édition, faisant «des tables des matières pour de grands ouvrages, des prospectus pour les libraires, muet d'ailleurs sur ses doctrines comme est muette une tombe sur les secrets de la mort» (p. 241-243). Balzac admire ce "gai bohémien $»^{7}$ et ses compagnons pour leur hauteur de vues, pour leur conduite fraternelle et pour l'envergure généreuse des projets que dissimule la modestie de leur vie présente.

Que la bohème soit ou non prometteuse, qu'elle prépare un avenir meilleur ou le fasse seulement et parfois indéfiniment attendre, la réponse n'allait pas de soi. Il y aura là pour Murger un problème personnel, fruit de son ambivalence : l'apologie de la pauvreté n'exclut pas chez lui la fascination pour l'argent, ni la vocation artistique le désir d'une position sociale. Pris entre sa sympathie pour ce milieu et son aspiration à s'établir, Murger ne put jamais sortir de la bohème, sans doute pour les motifs psychologiques et intimes qu'analyse Seigel (p. 53 sq.).

7 Cliché répandu depuis une célèbre chanson de Béranger (1828) : «Sorciers, bateleurs ou filous / Reste immonde / D'un ancien monde / Sorciers, bateleurs ou filous / Gais bohémiens, d'où venez-vous ?» 
Mais si tant d'autres littérateurs connurent eux aussi une bohème prolongée, c'est pour d'autres raisons encore: le bohème peut-il faire autre chose que pérenniser sa situation quand elle découle non d'un libre choix, mais d'une donnée structurelle? Cette question conduit à interroger la notion de marginalité bohémienne.

En octobre 1845, Champfleury publie le bref récit intitulé Chien-Caillou d'après le surnom du protagoniste. Fils d'un tanneur du Faubourg Saint-Marceau, le héros s'enfuit de chez lui à dix ans et devient graveur. Autodidacte, il n'a pas fréquenté d'atelier, acquérant son métier par l'étude solitaire des eaux-fortes de Rembrandt. "Sans éducation littéraire, sans éducation artistique, il était parvenu à faire des œuvres d'un grand sentiment. » Mais il tombe sous la coupe d'un marchand qui l'exploite sans scrupules et finit dans la misère. ChienCaillou est « de cette race de bohêmes qui restent toute leur vie bohêmes" (p.24). Il loue près de la place Maubert une mansarde dont Champfleury, promoteur du "réalisme » (mot nouveau en ce sens) et adepte d'une peinture véridique, dresse l'inventaire, complété d'une page ironique qui confronte sur deux colonnes « Les mansardes des poètes » et « Les mansardes réelles », nettement moins riantes.

Poésie et réalité s'opposent ici comme deux versions contrastées de la bohème. La première, lyrique, célèbre à la façon de Béranger les charmes de l'insouciance juvénile : « dans un grenier qu'on est bien à vingt ans" (Le Grenier). La deuxième, dramatique, dément cette vision positive. Dès La Peau de chagrin (1831), Balzac montre le pouvoir de l'argent et son corollaire, la mise à l'écart de la jeunesse instruite dans une société où priment l'utile et le lucratif. Raphaël de Valentin 
s'épuise dans sa mansarde à écrire un traité de philosophie qui passera inaperçu: l'auteur n'évoque même pas la sortie du livre, comme si le grand travail de Raphaël s'évaporait tout simplement. Illusions perdues relate l'odyssée du poète provincial sans fortune (Lucien) qui s'élance à l'assaut de la gloire littéraire, des élégances et de la richesse, sans connaître les lois d'une réussite durable et sans en posséder les moyens. L'écrivain moderne se veut libre, affranchi de la mendicité littéraire. Il compte vivre de sa plume et faire métier de son art. Mais s'il n'appartient pas à une famille aisée, il devra gaspiller son énergie dans les besognes mal payées. Ceux qui fréquentaient le groupe du Doyenné (1835-1836) avec Nerval étaient fils de grands bourgeois et de hauts fonctionnaires. La pauvreté se supporte mieux quand le présent s'adosse ainsi à des espérances d'héritage : "nous étions pauvres, mais aucun de nous n'eût consenti à s'attarder ou à se défaire la main dans le travail mercenaire », écrira Arsène Houssaye (p. 305). Par contre, les bohèmes des années 1840 ont presque tous des origines modestes. Le père de Murger est concierge-tailleur, celui de Champfleury secrétaire de mairie à Laon, celui de Charles Barbara marchand d'instruments de musique, celui d'Alfred Delvau tanneur au faubourg Saint-Marcel. Qu'il sorte du peuple ou de la petite bourgeoisie, le bohème intellectuel de cette génération pâtit de la «surqualification scolaire consécutive à la réforme de l'enseignement et à la fermeture des débouchés» (Heinich, p. 78). Artiste, il subit le divorce entre une image de soi «héroïque » (Grana, p. 57), fondée sur l'idée romantique de la vocation, et les conditions extérieures de son activité. Balzac, Champfleury et Sand incorporent ces données à la trame de leurs fictions. 


\section{Bohème idéalisée et bohémien réaliste : George Sand}

Inscrite dans la "préhistoire du bohémianisme", la double appellation bohème-bohémien pèse fortement sur le type littéraire et journalistique de l'artiste démuni, encore bohémien et pas encore tout à fait bohème au sens popularisé par Murger. L'œuvre de George Sand présente un intérêt spécial à ce stade. Des Lettres d'un voyageur (1834-1836) à Teverino (1845)8, ce type s'y rencontre plus d'une fois avec des constantes et des variations. Des rapports complexes unissent chez l'auteur trois éléments distincts, mais jamais tout à fait disjoints : la province de Bohême en pays tchèque (Consuelo-La Comtesse de Rudolstadt), une Bohème métaphorique désignée comme patrie spirituelle de l'artiste (Lettres d'un voyageur, La dernière Aldini, Teverino), enfin la bohème sociale de Paris (Horace), qui regroupe des étudiants et des artistes sans le sou.

Si la bohème vécue par l'écrivain en herbe (1831), bohème empirique avant la lettre, ne porte pas ce nom dans Histoire de ma vie (1854-1855), en revanche sa Bohème idéale s'édifie explicitement dans les années 1830 sur l'image du bohémien, célébré par les Lettres du "problématique voyageur » (1943, préface) ${ }^{9}$ et par La dernière Aldini. Toutefois la bohème «oubliée » de Sand ne s'arrête pas à cette période (voir Gould, p.357-365). Le bohémien revient assez souvent dans ses écrits postérieurs (romans, correspondance) pour avoir suscité des travaux spécifiques (Hecquet, p.103, et Genevray, 1998, p.39-51). Il reste à replacer leurs résultats

\footnotetext{
8 Cette date correspond à la parution des premières Scènes de la vie de Bohème de Murger, terminus ad quem choisi pour le présent article. Le thème bohémien se retrouvera chez Sand souvent associé au théâtre (Le Château des Désertes, Pierre-qui-roule).

${ }^{9}$ Préface (1843) des Lettres d'un voyageur.
} 
dans un cadre plus large que celui des études sandiennes et, notamment, dans l'histoire des représentations de l'artiste au XIXe siècle.

Ce personnage polymorphe traverse chez Sand divers sous-genres narratifs : l'autofiction (Lettres d'un voyageur), le récit d'aventures et sentimental (La dernière Aldini), le roman social (Horace), le roman historique de formation (Consuelo), la fantaisie (Teverino $)^{10}$. Plus fondamentalement, on distingue dans ce corpus deux variantes: d'une part, une bohème lointaine, située dans un pays étranger (La dernière Aldini, Consuelo, Teverino) où le cadre historique, peu circonstancié, ne pèse guère (sauf dans Consuelo); d'autre part, une bohème plus proche de l'auteur et du lecteur, inscrite dans la France contemporaine (Horace). Sand n'envisage pas le bohémien comme une somme de traits immuables. Elle remodèle plusieurs fois sa physionomie en fonction de ses besoins personnels, de son analyse de la société et des convictions qu'elle veut transmettre.

Le bohémien est d'abord, durant les années 1830, «fiction de soi » (voir Laforgue) et autodéfinition de l'écrivain. Aurore Dupin-Dudevant, alias George Sand, veut gagner par la plume son indépendance de femme. Le bohémien libertaire des Lettres d'un voyageur (1834-1836) lui fournit alors une identité de rechange : il habite une bohème idéale, autrement dit une idée de bohème qui légitime l'autonomie à la fois personnelle, professionnelle et intellectuelle de l'auteur. Sur le plan personnel, l'écrivain doit en effet gérer une situation anomique au regard des codes sexuels et matrimoniaux. La romancière

10 «Teverino est une pure fantaisie dont chaque lecteur peut tirer la conclusion qu'il lui plaira. » (Sand, 1852) 
revendique le statut d'artiste, mais la lettre VI (ÀÉverard) refuse de subordonner celui-ci à une utilité sociale ou à un engagement politique (voir Genevray, 2003). La liberté du voyageur, celle de "George le bohémien» (lettre de février 1836 à Frédéric Girerd) et de Lélio (La dernière Aldini), consiste à refuser l'enracinement géographique ainsi que les déterminations familiales ou sociales : «l'artiste, reprit Lélio, a pour patrie le monde entier, la grande Bohême, comme nous disons » (Sand, 1992a, p. 133). Il n'en va plus de même dans les textes ultérieurs, qui problématisent fortement l'indépendance de l'artiste et l'inscrivent dans certaines conditions particulières de temps et de lieu.

$\mathrm{Au}$ chapitre « Bohèmes » de son livre, Heinich (p. 32 et 41) évoque le basculement qui va de la bohème heureuse de Murger à celle, désespérée, de Champfleury ${ }^{11}$. Elle se garde pourtant de tracer une chronologie linéaire. En effet, outre que les Scènes de la vie de Bohème du premier commencent à paraître l'année même où le second publie Chien-Caillou (1845), ce basculement n'a rien d'un processus irréversible. Il s'agit plutôt d'un balancement entre deux versions simultanées. De même, il sera difficile de soutenir l'idée commode, mais trop simple qu'«avec Sand et Balzac, donc, la bohème change de signe » (Laisney, p. 299), passant du négatif au positif. Balzac, on l'a vu, présente les deux versions (Lucien, le Cénacle) et pose les deux signes. Quant à Sand, une telle idée ne vaut que si l'on s'en tient aux textes des années 1830. La formule inverse ne lui convient pas davantage si l'on se borne à dire qu'une bohème réaliste remplace sa bohème idéalisée. Heinich a donc le mot

11 L'appréciation de l'auteure concernant Murger ne fera pas l'unanimité. L. Chotard («Introduction» à Murger, p. 22) montre le pessimisme de la vision murgérienne. 
juste quand elle montre qu'Histoire de ma vie reflète chez Sand autobiographe « la tension entre ces deux acceptions - réaliste et idéalisée » (p. 80). L'extrait cité à l'appui relate les débuts de l'auteur, peu avant le succès d'Indiana (1832), qui inaugure sa carrière de romancière. La même tension se découvre dans ses fictions de l'artiste bohémien, Teverino par exemple ${ }^{12}$. Mais que faut-il entendre par idéaliser et ne devrait-on pas préciser le sens de réaliste?

L'idéalisation consiste à invoquer sous le nom de Bohème une contrée imaginaire, un espace mental, "patrie fantastique des âmes sans ambition et sans entraves »13. Elle présuppose une essence bohémienne (liberté d'esprit, d'allure et de mouvement, dons artistiques, marginalité sociale) affranchie des contingences extérieures. Mais la bohème des romans sandiens n'appartient pas toute à ce registre. Publié en feuilleton de novembre 1841 à mars 1842 dans la Revue indépendante, Horace perpétue accessoirement la mythologie du bohémien, crédité d'une "indépendance absolue » et d'une «fainéantise délicieuse » (p. 204), mais tend bien davantage vers un tableau réaliste de la bohème au sens murgérien, peuplée de jeunes Parisiens sans position et sans fortune. Le réalisme consiste à ancrer solidement ce milieu dans un temps et un lieu définis par des réalités d'ordre social, économique et politique. Aussi faut-il clarifier la terminologie. Il n'y a pas d'homologie de principe entre le couple «idéalisée vs réaliste » et le couple «enchantée vs désenchantée» donné pour

\footnotetext{
12 Heinich (p. 97) ne mentionne que Lélia (1833) et Elle et Lui (1859), sans rapport avec notre problématique.

13 Sand, 1970-1971, p. 817. Cette Bohême idéale s'apparente au Tyrol imaginaire de la Lettre I ou à la Suisse de la Lettre VII : " fonder, dans la belle Helvétie ou dans la verte Bohême, un colonie d'artistes » (p. 818).
} 
synonyme (Heinich, p. 37). Nous réservons la paire «idéaliste $v s$ réaliste " aux modes de figuration du bohémien, abstraction faite de la tonalité (optimiste ou pessimiste) et du jugement de valeur porté sur le personnage. Autrement dit, idéalisme et réalisme s'entendront ici comme catégories esthétiques dénotant un régime de représentation littéraire ${ }^{14}$, sans égard pour la qualification morale ou affective qui peut s'ajouter.

\section{Paris bohème : Horace}

L'action d'Horace a cours dans la période d'agitation populaire qui suit 1830. Elle inclut l'insurrection de juin 1832, qui fut réprimée dans le sang au cloître Saint-Merri. Sand choisit ses héros dans le quotidien : des étudiants (Horace et le narrateur), un bousingot (Jean Laravinière), une grisette (Eugénie), un rapin qui devient factotum de théâtre (Paul Arsène), une ouvrière qui monte sur les planches (Marthe). Paul et Marthe incarnent «la vocation vagabonde de l'artiste bohémien » (p. 276): au lieu de référer comme auparavant chez Sand à l'indépendance morale et spirituelle du peintre ou de l'acteur, le terme correspond cette fois à une catégorie d'artistes précaires, sans ressources, sans appuis et même sans formation. Fils de cordonnier et chargé de famille, Paul sacrifie sa passion et abandonne la peinture, qui rapporte trop peu pour faire vivre les siens. L'auteur en fait un représentant typique du Paris laborieux : «c'était le type peuple incarné dans un individu; non le peuple robuste et paisible qui cultive la terre, mais le peuple artisan, chétif, hardi, intelligent et alerte » (p. 48).

\footnotetext{
14 «Le réalisme sérieux des temps modernes ne peut représenter l'homme autrement qu'engagé dans une réalité politique, économique et sociale en constante évolution. » (Auerbach, p. 459)
} 
Balzac et Sand rapportent la condition du bohémienbohème aux changements qui affectent la France contemporaine. À l'essor des couches moyenne et supérieure de la bourgeoisie sous la monarchie de Juillet répond, dans Horace, la frustration des milieux populaires et de la petite bourgeoisie ${ }^{15}$. Ce roman (sans doute le plus balzacien de Sand) prend acte de la situation concrète de l'artiste dans la société du temps. La marchandisation déjà évoquée n'en est qu'un aspect, pourvu d'ailleurs d'un double tranchant. Sur ce thème, L'Artiste a publié dès 1831-1832 deux analyses différentes. L'une, citée plus haut (voir note 6), regrette ses effets dommageables. L'autre indique des suites bénéfiques, car le passage du mécénat privé ou de la commande étatique au marché de la librairie, combiné avec la diffusion croissante de l'imprimé et de la culture, peut avoir des résultats positifs pour l'artiste moderne. Tirant ses revenus d'un travail mis par lui-même en vente, il s'affranchit de la sujétion morale ou esthétique qui le pliait à la volonté des grands. Le voici libre. Non point dans l'absolu, libre plutôt de choisir ses affiliations, de lier son sort moral et matériel à d'autres qu'aux privilégiés: "l'artiste aujourd'hui est placé au milieu de la société tout entière, il s'inspire des désirs et des souffrances de tous, il parle à tous, il crée pour tous; il n'est plus valet mais peuple; il n'attend son salaire que de son travail et des productions libres de son génie; sa position sociale est donc devenue plus morale, plus indépendante, plus capable de favoriser les progrès de l'art. » (Saint-C, p. 53) Dans les termes que nous soulignons s'ébauche

\footnotetext{
15 À « la bourgeoisie encroûtée qui, maîtresse de toutes les forces de l'État, en fait un misérable trafic » (1982, p. 68), appelée aussi «le juste-milieu et la bourgeoisie parvenue» (p. 109), Sand oppose « la jeune bourgeoisie » (p. 69) qu'elle estime capable d'embrasser la cause démocratique et républicaine.
} 
la double postulation de l'artiste sandien: sans attaches et jaloux de sa liberté, même s'il parle au nom du peuple (Teverino), ou engagé aux côtés des petits et des opprimés (Consuelo).

L'artiste, chez Sand, se fait peuple ou porte-parole de celui-ci en vertu d'une solidarité objective fondée sur les conditions matérielles et morales imparties aux créateurs. Cet infléchissement caractérise tous ses personnages de bohémiens depuis le début des années 1840 et leur donne une couleur politique qu'on ne trouvera pas chez le bohème de Murger, qui " se désintéresse totalement des affaires de la cité » (Laisney, p. 303). Cette couleur imprégnait déjà La dernière Aldini, mais le cadre restait là assez vague, de même que l'analyse des forces en présence. La «Bohême» idéale de Lélio gommait les distinctions de classe ${ }^{16}$, celle d'Horace les souligne et marque qu'il faut choisir son camp, quitte à nouer des alliances. Paul Arsène, « républicain populaire » (Sand, 1982, p. 181), participe aux barricades de 1830 parce que «mieux vaut mourir d'un coup de fusil que de faim» (p.54). Laravinière, "étudiant émeutier» (p. 70) salué «président des bousingots» (p. 68), veut révolutionner le nouveau régime « en faveur du peuple» (p. 205). Luttant contre «la puissance bourgeoise» (p. 189), il choisit cette cause par conviction et non par intérêt: «Je suis très content de la société en ce qui me concerne. J'y jouis d'une indépendance absolue, et j'y savoure une fainéantise délicieuse. Je la traverse en véritable bohémien, et je n'y ai qu'une affaire, qui est de conspirer pour son renversement; car le peuple souffre [...]. » (p. 204)

\footnotetext{
16 Gould (p. 360) la définit comme « a socially liberated space that erases the confines of race, gender, nation and especially class ».
} 
Sand joue sur l'articulation entre artiste ambulant (sa définition minimale du bohémien) et artiste prolétaire (soumis à une grande précarité) pour promouvoir l'artiste pauvre ${ }^{17}$. Ceci revient à l'extraire de la marge : ou le bohémien sort du peuple, comme Marthe et Paul, ou il s'agrège au peuple (Consuelo), fût-il comme Teverino originaire (à demi et par simple hypothèse) d'une noble famille. Un tel parti conduit à épurer le type bohémien pour le délivrer des stigmates dégradants de sa marginalité première ${ }^{18}$. La mère de Consuelo, zingara misérable, devait mendier: sans renier ouvertement la condition maternelle, l'héroïne s'en libère et construit sa vocation d'artiste comme un véritable métier, qui la hisse sur les scènes les mieux cotées d'Europe. Euphémisant toujours plus l'image d'abord peu positive de la bohémienne, le diptyque finit par la sublimer dans l'épilogue quand, tournant le dos à sa brillante carrière, Consuelo part mener une vie errante et obscure parmi le peuple de Bohême (La comtesse de Rudolstadt, 1844).

Ainsi le bohémien fictif se détache-t-il de l'auteur, qui s'était approprié le mythe pour légitimer ses choix personnels. $\mathrm{Au}$ bohémien libertaire (le voyageur, Lélio) succède ce bohémien solidaire qu'est l'artiste pauvre. Sand ne le dépeint pas pour lui-même, comme objet d'une esquisse naturaliste ou satirique de "physiologie » sociale à la manière du temps. Elle le charge d'incarner son idéal philosophico-politique d'égalité républicaine, et c'est dans cette autre acception qu'on peut

17 Elle s'y emploie aussi dans la vie réelle, conseillant le maçon-poète Charles Poncy et ouvrant aux poètes ouvriers les colonnes de la Revue indépendante (1842).

18 « Ma mère était plus bas placée que la vôtre [...]. Elle n'appartenait pas à cette classe laborieuse et persévérante qui vous donne à vous un titre de noblesse dans le peuple. Elle était de la race vagabonde et avilie des Bohémiens de ce monde. », écrit Sand à Ch. Poncy (23 décembre 1843). 
parler d'idéalisme. L'artiste pauvre fait siennes des aspirations démocratiques qui dépassent l'enjeu de son propre statut: Sand l'érige en porteur des revendications populaires, qu'il s'agisse des petites gens (Horace) ou d'un peuple opprimé, nié comme nationalité (la Bohême de Consuelo) ${ }^{19}$.

L'évolution ainsi marquée n'est d'ailleurs pas linéaire, car dans Teverino revient en force la première version du bohémien. Le héros éponyme semble conçu pour incarner une fois encore la Bohème métaphorique des années 1830: il n'a aucune place marquée dans l'espace social, aucun engagement durable, et son patriotisme italien reste platonique. Simultanément, l'auteur réactive les connotations défavorables du terme. "Poète naturel ", bon chanteur, doué pour la comédie, Teverino répugne par indolence à perfectionner ses dons et reste un dilettante. Bien qu'elle le peigne avec sympathie, Sand ne s'identifie pas sans réserve à ce bohémien qu'elle range dans la catégorie des « aventuriers » (1992b, p. 628, 643, 674, et 1852) et dont elle fait un homme seul. Au contraire, les bohèmes se regroupent pour se soutenir mutuellement en réunissant leurs espoirs et leurs expédients: ainsi se forment, non point en marge ou à la périphérie, bien plutôt dans les interstices de l'officialité littéraire et culturelle, ces unions, groupes, cénacles, fraternités et colonies d'artistes fondés sur l'entraide.

Si Marthe et Paul Arsène optent pour «la vocation vagabonde de l'artiste bohémien », c'est qu'ils ne peuvent être artistes tout court, avec la formation, le loisir, les ressources et les relations nécessaires. Paul Arsène engloutit sa jeunesse dans les besognes alimentaires :

19 Incarnée notamment par Zdenko, « l'artiste vagabond, qui est mon égal et mon confrère », dit Consuelo. 
Il fallait donc qu'il allât grossir le nombre, énorme déjà, des enfants perdus de cette civilisation égoïste qui a oublié de trouver l'emploi des pauvres maladifs et intelligents. À ceux-là le théâtre, la littérature, les arts, dans tous leurs détails brillants ou misérables, offrent du moins une carrière, où, par malheur, beaucoup se précipitent par mollesse, par vanité ou par amour du désordre, mais où, en général, le talent et le zèle ont des chances d'avenir. » (Sand, 1982, p. 277)

La marginalité passe pour inséparable des définitions usuelles de la bohème (Heinich, p. 28 et 35). Pourtant, Murger ne voit dans «la grande famille des artistes pauvres, fatalement condamnés à la loi de l'incognito » et vivant " pour ainsi dire en marge de la société, dans l'isolement et dans l'inertie »» qu'une sous-catégorie, une "Bohème ignorée » distincte de «la vraie Bohème » «qui fait en partie le sujet » de son livre ( "Préface », p. 35 et 40). Dès lors qu'on voit en elle, comme Sand, une « carrière » non dénuée de « chances d'avenir », la bohème n'est pas marginale sur le plan numérique. Manifestation exemplaire d'une situation répandue, elle offre une voie de secours à une catégorie nombreuse, enfants des couches modestes sans débouchés pour leurs aptitudes : «il est certain que le domaine de l'art était le seul où ils pussent trouver un refuge pour leur existence matérielle, un milieu pour le développement de leur vie intellectuelle » (Sand, 1982, p. 276). Quant à savoir s'il s'agit d'une forme temporaire d'apprentissage ou d'une relégation à vie dans ce qu'on nomme aujourd'hui les "petits boulots», les formules employées par Sand hésitent à trancher : il est trop tôt pour préjuger de l'avenir.

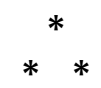


George Sand ne connaît qu'un seul terme pour désigner le bohémien des chemins et celui de la grande ville. Support d'identification personnelle dans sa correspondance et dans les Lettres d'un voyageur ou personnage de roman dans La dernière Aldini, Horace, Consuelo et Teverino, la figure du bohémien circule à travers ses écrits des années 1835-1845 entre plusieurs espaces: Bohême géographico-historique, patrie imaginaire des artistes, bohème sociale parisienne. Tout en soulignant la polyvalence du vocable, nous avons mis l'accent sur Horace comme jalon méconnu dans la préhistoire du bohémianisme. Là émerge en effet l'artiste-bohème qui prendra la vedette chez Murger. Toutefois, la bohème d'Horace ne coïncide pas avec la bohème «fauchée et heureuse » que l'on a cru rencontrer chez ce dernier (voir Heinich, p. 28). Ce n'est pas pour autant la bohème désolante du génie obscur et exploité que dépeint Champfleury dans Chien-Caillou. Non que Sand cherche un moyen terme entre ces deux pôles. Elle ne valorise pas une marginalité censément héroïque, pas plus qu'elle ne la disqualifie comme voie sans issue. Son propos consiste à faire le point sur un état social dont la marginalisation des créateurs offre un symptôme parmi d'autres. Si l'auteur de Consuelo et surtout de Teverino continue de chérir la figure presque intemporelle de l'artiste ambulant, le bohémien d'Horace "se parisianise $»^{20}$. Le cercle de ses amitiés appartient au prolétariat intellectuel urbain et son expérience individuelle s'inscrit dans un cadre historique nettement défini: la Révolution de juillet a été confisquée par la bourgeoisie moyenne et supérieure, les talents éclos dans le petit peuple ont peu d'avenir. Qu'il y ait dans l'existence de la bohème ainsi

\footnotetext{
${ }^{20}$ Loïc Chotard, « Introduction » à Murger (p. 7).
} 
particularisée un « vivant reproche à la vie bourgeoise » (Seigel, p. 68) devient de plus en plus clair vers la fin des années 1840. Ouverte ou souterraine, la politisation de la bohème découle de ce diagnostic. C'est pourtant l'époque où les sympathies radicales de Murger se refroidissent. Aussi revient-il à Sand d'avoir donné la préfiguration romanesque d'une convergence idéologique et pratique entre bohème et révolution qui ne se cherchera dans les faits qu'à partir de 1848.

\section{Bibliographie}

AuERBACH, Erich. (1977), Mimesis, Paris, Gallimard, coll. « Tel »

BALZAC, Honoré de. (1990), Illusions perdues, Paris, Flammarion, coll. « GF».

Champfleury. (1990), Chien-Caillou. Fantaisies d'hiver, Paris, Éd. des Cendres.

Delord, Taxile, Arnould Frémy et Edmond TeXIER (1854), ParisBohème, Paris, A. Taride.

D'Ennery, Adolphe et Grangé. (1843), Les Bohémiens de Paris, drame en 5 actes et 8 tableaux donné à l'Ambigu-Comique le 27 septembre 1843, Paris, Marchant.

GENEVRAY, Françoise. (1998) "Des Lettres d'un voyageur à Teverino: George Sand ou le voyageur sans bagage», Romantisme, $\mathrm{n}^{\circ} 99$. 
—. (2003), «Bohême, bohème, bohémien : autour de George Sand », journée d'études "L'Auteur en marge », 10 avril, $<$ http://www.univ-lyon3.fr> (onglets: Recherches / Ressources/Articles).

GoulD, Evelyn. (1998), "George Sand's forgotten Bohemia », dans David A. Powell et Shira Malkin (dir.), Le Siècle de George Sand, Amsterdam, Rodopi.

Goulemot, Jean-M. et Daniel Oster. (1992), Gens de lettres, écrivains et bohèmes: l'imaginaire littéraire, 1630-1900, Paris, Minerve.

GrANA, César. (1964), Bohemian Versus Bourgeois: French Society and the French Man of Letters in the Nineteenth Century, New York-Londres, Basic Books.

HECQUET, Michèle. (1992), "Enfants de Bohême : naissance et légitimation chez Sand», Revue des sciences humaines, $\mathrm{n}^{\circ} 226$.

HEINICH, Nathalie. (2005), L'Élite artiste. Excellence et singularité en régime démocratique, Paris, Gallimard.

HouSSAYE, Arsène. (1971), "La Bohème romantique », dans Les confessions. Souvenirs d'un demi-siècle, 1830-1880, t. 1, Genève, Slatkine Reprints (réimpr de l'éd. de Paris, 1885-1891).

LAFORGUE, Pierre. (2003), Corambé. Identité et fiction de soi chez George Sand, Paris, Klincksieck.

LAISNEY, Vincent. (2008), «De la sociabilité bohémienne à la sociabilité cénaculaire (les Scènes de la vie de bohème de Henry Murger) », dans Sarga Moussa (dir.), Le Mythe des Bohémiens dans la littérature et dans les arts en Europe, Paris, L'Harmattan. 
Murger, Henry. (1988), Scènes de la vie de bohème, Paris, Gallimard, coll. « Folio classique ».

SAINT-C [Alexandre de St-Chéron]. (1832), « De la position sociale des artistes », L'Artiste, $\mathrm{n}^{\circ} \mathrm{IV} / 5, \mathrm{p} .53$.

SAND, George. (1992a), La dernière Aldini, dans Vies d'artistes, Paris, Presses de la Cité, coll. « Omnibus ».

-. (1992b), Teverino, dans Vies d'artistes, op. cit.

—. (1982), Horace, Meylan, Éd. de l'Aurore.

—. (1852), Notice précédant la réédition de Teverino.

-. (1970-1971), Lettres d'un voyageur, dans OEuvres autobiographiques, Gallimard, coll. "Bibliothèque de la Pléiade », t. II.

SEIGEL, Jerrold. (1991), Paris bohème. Culture et politique aux marges de la vie bourgeoise, 1830-1930, trad. fr. par O. Guitard, Paris, Gallimard, [New York, 1986].

La Bohême Galante (1852), Les Petits Châteaux de Bohême (1853), Promenades et Souvenirs (1854-1855).

\section{Résumé}

L'étude de la bohème est une page importante dans l'histoire des représentations de l'artiste au XIX ${ }^{\mathrm{e}}$ siècle. La décennie considérée dans cet article correspond à l'intervalle séparant l'émergence de la bohème comme motif littéraire et sa consécration définitive par les feuilletons de Henry Murger (1845-1849). La double appellation bohème / bohémien pèse encore fortement à ce stade sur la figure journalistique et 
littéraire de l'artiste pauvre ou de l'intellectuel démuni que l'on rencontre chez Balzac (Illusions perdues) et Champfleury (Chien-Caillou). Cependant, les travaux sur la bohème (J. Seigel, N. Heinich, A. Glinoer) s'attachent peu aux multiples textes de Sand, qui, des Lettres d'un voyageur (1834-1836) à Teverino (1845) en passant par Consuelo et Ladernière Aldini, intéressent cette phase initiale du bohémianisme. La fin de l'article met l'accent sur Horace (1842), roman où la figure mythique, presque intemporelle, $\mathrm{du}$ bohémien, artiste indépendant et vagabond, fait place à l'analyse d'une situation historique et d'un état social contraignants, qui n'offrent guère d'avenir aux talents créateurs d'origine populaire.

\section{Abstract}

The study of bohème is an important chapter in the history of nineteenth-century representations of the artist. The decade under scrutiny in the present article corresponds to the interval between the emergence of bohème as a literary motif and its definitive consecration through Henry Murger's feuilletons (1845-1849). The dual designation as bohème/bohemién still bears heavily, at this point, on the journalistic and literary figure of the penniless artist or the destitute intellectual as portrayed by Balzac (Illusions perdues) and Champfleury (Chien-Caillou). Research work on bohème (J. Seigel, N. Heinich, A. Glinoer), however, takes little account of Sand's numerous texts which, from Lettres d'un voyageur (1834-1836) to Teverino (1845) including Consuelo and Ladernière Aldini, belong to this initial phase of bohemianism. The end of the article focuses on Horace (1842), a novel where the mythical, almost timeless figure of the bohemian as an independent, wandering artist gives way to an analysis of the constraints imposed by a historical situation and social conditions that offer very little scope for a promising future to creative talents of humble birth. 